\title{
BUFFERING EFFECT FOR 2ND COVID-19 LOCKDOWN: THE ROLE OF ACADEMIC E-LEARNING ADOPTION AMONG GENERATION Z
}

\author{
Jessica Ranieri, Federica Guerra, \& Dina Di Giacomo \\ Department of Life Health and Environmental Sciences, University of L'Aquila (Italy)
}

\begin{abstract}
Background. The undergraduate community is composed of Generation $\mathrm{Z}$ members who constitute a social generation of digital natives who are technologically skilled. Their widespread exposure to technology accounts for their comfort with and strong knowledge of digital media. The government adoption of e-learning in academic education during the coronavirus disease (COVID-19) pandemic may be beneficial to such digitally skilled individuals.

Some studies have underscored the e-learning adoption adverse psychological impact on the mental health of the younger generation. In fact, the findings underscore an increase in psychological distress, excessive fear of infection, pervasive anxiety, frustration and boredom, a high level of stress, and post-traumatic stress symptoms.

We aimed to detect the protective factor for academic community during social restriction for pandemic in 2nd Italian lockdown analysing the adaptive behaviour of undergraduate in 3 field panels of academic education (life sciences, physical and engineer sciences, human and social sciences). We aimed to determine the psychological impact of prolonged e-learning on emotional regulation among undergraduate students. A secondary objective was to identify key components for preventive interventions targeted toward the academic community by investigating the buffering effect of e-learning in academic education on exposure to the pandemic.

Methods. An online cross-sectional survey was conducted on 570 university students (aged 18-26 years) pursing degrees in life sciences, physical and engineering sciences, and social sciences in Italy. They were recruited using snowball sampling. We administered emotional (PDEQ, CSSQ, CAS), personality traits (BFI-10) and affinity for e-learning (AEQ) measures.

Results. Our findings suggest that a majority of the university students developed peritraumatic dissociative experience and stress, but not dysfunctional coronavirus anxiety during the 2nd COVID-19 lockdown in Italy. Nevertheless, the present findings also highlight the fragility of younger Gen $\mathrm{Z}$ undergraduate students who are beginning their academic journey amid the COVID-19 pandemic. Further, coronavirus distress significantly predicted mental health through the mediating effect of personality traits and e-learning affinity.

Conclusions. Therefore, health care professionals are encouraged to implement psychological support interventions that strengthen one's ability to manage stressful situations and reinforce their status as a digital native. Consequently, they may realize the power of their personal strengths, which in turn may mitigate their stress and peritraumatic dissociative experience when they deal with challenges, enhance their competence, and enable them to adopt effective coping strategies.
\end{abstract}

Keywords: COVID-19, generation Z, buffering effect, e-learning, university students.

\section{Introduction}

During the COVID-19 pandemic, many countries decided to close all learning institutions including universities to prevent the spread of coronavirus. The COVID-19 pandemic has had a catalytic effect on the changes in educational processes worldwide. It caused an abrupt shift from face-to-face to online classes ${ }^{1}$. Several studies showed a psychological impact of the Covid-19 emergency on college students. Stress significantly decreases learning and negatively affects psychological well-being of students ${ }^{2}$. However, few studies have took into account the features inherent the $Z$ Generation ${ }^{3}$.

Gen $\mathrm{Z}$ defines the current college population. Indeed, Generation $\mathrm{Z}$ constitutes agile and active generation in the digital online environment which is smartphone natives savvy with mobile technology ${ }^{4}$. 
The massive adoption of e-learning in academic education during the pandemic may have been a positive change for digitally skilled individuals, such as Gen $\mathrm{Z}$ members. Our aim was to investigate the affinity for e-learning as a resilience strategy for Gen Z during Covid-19.

\section{Design}

An online cross-sectional survey was conducted in December 2020 among university students pursing degrees in life sciences, physical and engineering sciences, and social sciences in Italy. During this period, the total confirmed cases of COVID-19 was 1.870 .576 in Italy ${ }^{5}$. They were recruited from a dedicated university community using social media platforms using snowball sampling. Data were collected anonymously.

\section{Objectives}

Aim of the present study was to detect the protective factor for academic community during social restriction for pandemic in 2nd Italian lockdown (December 2020) analysing the adaptive behaviour of undergraduate in 3 field panels of academic education (life sciences, physical and engineer sciences, human and social sciences). We aimed to determine the psychological impact of prolonged e-learning on emotional regulation among undergraduate students. A secondary objective was to identify key components for preventive interventions targeted toward the academic community by investigating the buffering effect of e-learning in academic education on exposure to the pandemic.

\section{Methods}

\subsection{Participants}

The participants were 570 Italian undergraduate students aged 18-26 years old (mean age $=22.2$, $\mathrm{SD} \pm 2.32$ ). Demographic characteristics of the participants: $74 \%$ (n.422) of them were females, and $26 \%$ (n.158) males; 4.4\% were living in Northern (n.25), 16.1\% Central (n.92), and 79.5\% Southern Italy (n.453). The inclusion criteria were as follows: (a) aged 18-26 years, (b) an undergraduate student, and (c) provision of informed consent.

\subsection{Outcomes and covariates}

The socio-demographic data were assessed using a socio-demographic form. Psychological battery has been composed of n.5 self-reports evaluating anxiety symptoms, personality traits, peritraumatic dissociation, and stress to measure the presence/absence of psychological symptoms and related severity. An ad hoc questionnaire was used to assess e-learning affinity.

Coronavirus Anxiety Scale (CAS) ${ }^{6}$. The CAS is a brief 5-item mental health screening tool that can be used to detect dysfunctional anxiety associated with the COVID-19 crisis. Specifically, it assesses the cognitive, behavioural, emotional, and physiological dimensions of coronavirus anxiety. Each item is rated on a 5-point scale that ranges from 0 (not at all) to 4 (nearly every day), and these ratings indicate the frequency with which the symptom has been experienced during the past two weeks. A total score $\geq 9$ is indicative of dysfunctional anxiety.

Big Five Inventory-10 (BFI-10) ${ }^{7}$. The BFI-10 evaluates the five personality dimensions on a 5-point scale ranging from 1 (strongly disagree) to 5 (strongly agree), each with two items: OPenness (OP), COnscientiousness (CO), Emotional Stability (ES), EXtraversion (EX), and AGreeableness (AG).

Peritraumatic Dissociative Experiences Questionnaire (PDEQ) ${ }^{8}$. It is a 10 -items self-report questionnaire measuring peritraumatic dissociation. The PDEQ has well-established psychometric properties, with higher total scores indicating increased peritraumatic dissociation. A score above 15 is indicative of significant dissociation.

COVID-19 Student Stress Questionnaire (CSSQ) ${ }^{9}$. The CSSQ measures COVID-19-related sources of stress among undergraduate students. It consists of 7 items, which are rated on a 5-point scale that ranges from zero ('Not at all stressful') to four ('Extremely stressful'). The CSSQ consists of the following three subscales, which assess COVID-19-related stressors among students: Relationships and academic life; Isolation; and Fear of contagion. This scale also yields a global stress score, which can range from 0 to 28 .

Affinity for e-learning Questionnaire (AEQ). The AEQ is an experimental self-report measure that assesses affinity for e-learning. It is composed of 10 items, which are rated on a five-point Likert scale that ranges from 'Completely disagree' to 'Completely agree'. It assesses self-confidence in relation to service access, convenience and flexibility, lesson attendance, involvement, and information 
technology skills. A pilot study was conducted using a sample drawn from the target population (not included in this study) to examine the reliability of the AEQ. The internal consistency of the scale was high $(\alpha=0.8)$.

\subsection{Statistical analyses}

The data analysis was performed using Jamovi statistical software, with a fixed $\alpha$-value $\leq 0.05$. All demographic data were analyzed and presented as number $(\mathrm{N})$ and percentage $(\%)$. Using ANOVA test as appropriate, we compared emotional severity by demographic variables. Pearson's correlation analysis was conducted to examine the relationships between the study variables.

\section{Results}

The mean age of the participants (n. 570) was 22.2 years ( $\mathrm{SD}=2.32$, range: $18-26)$, and $74 \%$ of them were women. A majority of the participants were pursuing a bachelor's degree $(55,1 \%)$. Moreover, $62,3 \%$ of the participants were off-campus students, and $41,4 \%$ (n.236) belonged with scientific academic discipline.

A considerable part of the sample (86,5\%) showed significant peritraumatic dissociative experience (PDEQ) and 62,3\% resulted in high level stressed emotional condition. 29,6\% showed coronavirus anxiety. For dimensions of personality status, the prevalence for each traits were: $47,7 \%$ moderate level of conscientiousness (47,2\% high, 5.1\% low level); 78,4\% moderate level of emotional stability $(12,6 \%$ high and 8,9\% low level); 48,6\% moderate level of openness $(42,5 \%$ high, $8,9 \%$ low level); 53,2\% moderate extroversion level (25,3\% low, $21,6 \%$ high level); then, $65,6 \%$ moderate level of agreeableness (22,3\% high, $12,1 \%$ low level).

We conducted comparative analyses using grouped data. The participants were divided into two groups based on their median age (22 years): younger Gen $\mathrm{Z}$ group $(\mathrm{n}=305)$ and older Gen $\mathrm{Z}$ group $(n=265)$. One-way ANOVA was conducted to examine age differences in emotional variables. These group comparisons revealed that there was a significant difference between the younger and older Gen $\mathrm{Z}$ groups in PDEQ $(\mathrm{p}=0.002)$ and in AEQ $(\mathrm{p}<.001)$. Specifically, high peritraumatic dissociation and lower affinity e-learning levels were reported by the younger Gen $\mathrm{Z}$ group.

Then, the participants were divided into three groups based on their academic education field: Life Sciences (LS) group ( $\mathrm{n}=143)$, Physical and Engineer Sciences (PES) group $(\mathrm{n}=236)$, and Human and Social Sciences (HSS) group $(\mathrm{n}=191)$. One-way ANOVA was conducted to examine field panel differences in emotional and personality variables. These group comparisons revealed that there was a significant difference between HSS and LS groups on Extroversion (BFE), HSS reported high extroversion personality trait $(\mathrm{p}=0.02)$; between HSS, LS and PES groups on Isolation (CIS), HSS and PES reported high isolation ( $\mathrm{p}=0.007)$; and between HHS and LS groups on Coronavirus Anxiety (CAS) $(\mathrm{p}=0.001)$, high Anxiety levels were reported by HHS group (see Table 1).

Table 1. Raw score of the sample groups on psychological assessment.

\begin{tabular}{|c|c|c|c|c|c|c|c|c|c|c|c|c|}
\hline Tests/Groups & \multicolumn{6}{|c|}{ Young } & \multicolumn{6}{|c|}{ Old } \\
\hline & \multicolumn{2}{|c|}{$\begin{array}{c}\text { Life } \\
\text { sciences } \\
(n=83)\end{array}$} & \multicolumn{2}{|c|}{$\begin{array}{c}\text { Physical\&en } \\
\text { gineer } \\
\text { sciences } \\
(\mathrm{n}=141)\end{array}$} & \multicolumn{2}{|c|}{$\begin{array}{l}\text { Human\&so } \\
\text { cial sciences } \\
\quad(\mathrm{n}=81)\end{array}$} & \multicolumn{2}{|c|}{$\begin{array}{c}\text { Life } \\
\text { sciences } \\
(n=60)\end{array}$} & \multicolumn{2}{|c|}{$\begin{array}{l}\text { Physical\&en } \\
\text { gineer } \\
\text { sciences } \\
(\mathrm{n}=95) \\
\end{array}$} & \multicolumn{2}{|c|}{$\begin{array}{c}\text { Human\&so } \\
\text { cial sciences } \\
(\mathrm{n}=110)\end{array}$} \\
\hline & $\mathrm{X}$ & SD & $\mathrm{X}$ & SD & $\mathrm{X}$ & $\mathrm{SD}$ & $X$ & SD & $\mathrm{X}$ & SD & $\mathrm{X}$ & SD \\
\hline PDEQ & \multicolumn{2}{|c|}{$27.4 \pm 10.1$} & \multicolumn{2}{|c|}{$30.1 \pm 9.82$} & \multicolumn{2}{|c|}{$28.1 \pm 10.5$} & \multicolumn{2}{|c|}{$24.6 \pm 10.3$} & \multicolumn{2}{|c|}{$26.1 \pm 9.94$} & \multicolumn{2}{|c|}{$27.3 \pm 10.00$} \\
\hline \multicolumn{13}{|l|}{ BFI } \\
\hline $\mathrm{Ag}$ & \multicolumn{2}{|c|}{$6.27 \pm 1.48$} & \multicolumn{2}{|c|}{$6.41 \pm 1.51$} & \multicolumn{2}{|c|}{$6.23 \pm 1.69$} & \multicolumn{2}{|c|}{$6.18 \pm 1.60$} & \multicolumn{2}{|c|}{$6.03 \pm 1.57$} & \multicolumn{2}{|c|}{$6.10 \pm 1.61$} \\
\hline $\mathrm{Co}$ & \multicolumn{2}{|c|}{$6.96 \pm 1.80$} & \multicolumn{2}{|c|}{$7.33 \pm 1.46$} & \multicolumn{2}{|c|}{$7.41 \pm 1.72$} & \multicolumn{2}{|c|}{$7.57 \pm 1.61$} & \multicolumn{2}{|c|}{$7.42 \pm 1.55$} & \multicolumn{2}{|c|}{$7.27 \pm 1.77$} \\
\hline ES & \multirow{2}{*}{\multicolumn{2}{|c|}{$6.20 \pm 1.47$}} & \multicolumn{2}{|c|}{$6.27 \pm 1.30$} & \multicolumn{2}{|c|}{$6.00 \pm 1.21$} & \multicolumn{2}{|c|}{$6.02 \pm 1.30$} & \multicolumn{2}{|c|}{$6.14 \pm 1.15$} & \multicolumn{2}{|c|}{$6.09 \pm 1.23$} \\
\hline Op & $7.19 \pm 1.82$ & & 7.0 & 1.81 & & 1.96 & 7.1 & 2.04 & & \pm 1.99 & & \pm 2.03 \\
\hline Ex & & \pm 2.11 & 5.9 & 2.06 & & 1.82 & 5.5 & 2.02 & & \pm 1.68 & & \pm 2.05 \\
\hline CSSQ & & \pm 5.92 & 17. & 4.89 & & 5.35 & 16. & $=5.52$ & & \pm 6.22 & & \pm 5.23 \\
\hline CRA & & \pm 3.89 & 8.7 & 3.30 & & 3.74 & 8.8 & $=3.56$ & & \pm 4.05 & & \pm 3.76 \\
\hline CIS & & \pm 2.16 & 5.5 & 1.99 & & 2.19 & 4.4 & 2.25 & & \pm 2.26 & & \pm 1.89 \\
\hline $\mathrm{CFC}$ & & \pm 1.15 & 2.9 & 0.98 & & 0.95 & 2.6 & 1.12 & & \pm 1.27 & & \pm 1.05 \\
\hline AEQ & & \pm 9.54 & 28. & 7.90 & & 8.90 & 30 . & 9.65 & & \pm 9.91 & & \pm 9.94 \\
\hline CAS & & \pm 5.39 & 6.2 & 4.40 & & 5.04 & 3.8 & 3.79 & & \pm 4.97 & & \pm 5.40 \\
\hline
\end{tabular}


Finally, we examined the correlations between e-learning affinity, emotional indices and personality traits. Pearson's correlation analysis revealed that there was a negative relationship between scores on the PDEQ and Consciousness (BFI) $(r=-0.09 ; \mathrm{p}=0.02)$, and affinity e-learning $(\mathrm{r}=-0.42$; $\mathrm{p}<.001)$; a positive relationship between PDEQ and CAS anxiety score $(\mathrm{r}=0.64 ; \mathrm{p}<.001)$ and CSSQ CRA, CIS and CFC $(\mathrm{p}=<.001)$. There was a negative relationship between Agreeableness (BFI) and CSSQ CRA ( $\mathrm{r}=-0.08 ; \mathrm{p}=0.04)$. There was a positive relationship between Consciousness (BFI) and affinity e-learning (AEQ) $(r=0.11 ; \mathrm{p}=0.007)$ and a negative relationship with CSSQ CRA $(\mathrm{p}=0.001)$ and CIS $(\mathrm{p}=0.02)$. There was a negative relationship between Emotional Stability (BFI) and AEQ and CFC $(\mathrm{p}=0.02)$. There was a positive relationship between Extroversion (BFI) and Isolation (CIS) $(\mathrm{r}=0.12 ; \mathrm{p}=0.002)$. There was a positive relationship between Openness (BFI) and coronavirus anxiety $(\mathrm{CAS})(\mathrm{r}=0.08 ; \mathrm{p}=0.04)$ and Relationship and academic life (CSSQ) $(\mathrm{r}=0.08 ; \mathrm{p}=0.03)$. There was a negative relationship between anxiety (CAS) and AEQ $(r=-0.22 ; \mathrm{p}<.001)$ (see Figure 1).

Figure 1. Correlation matrix partial plot.

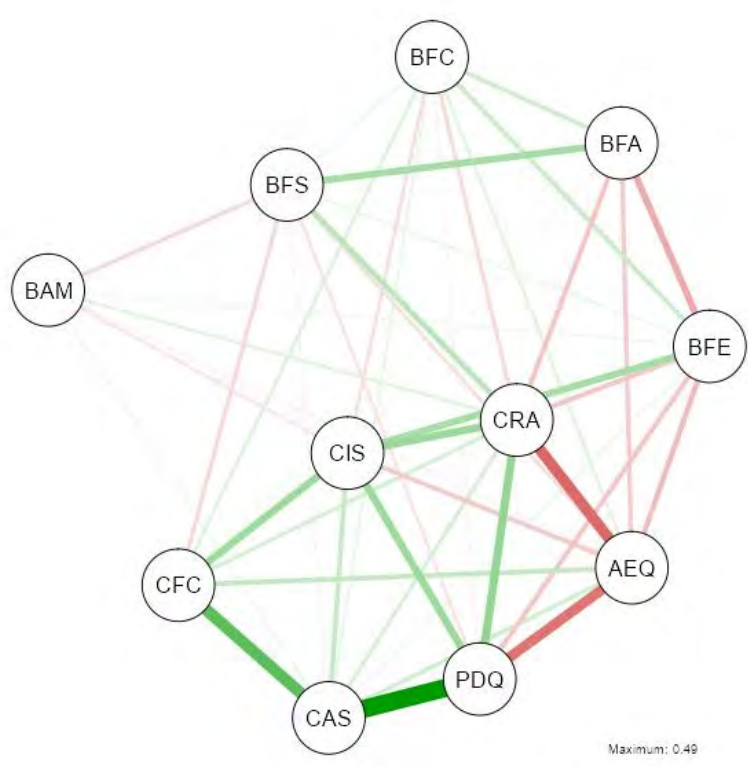

\section{Discussion}

This cross-sectional study was focused on protective factors for Gen Z community during prolonged academic e-learning for pandemic in 2nd Italian lockdown (December 2020); we wanted to investigate the e-learning affinity as a protective factor for traumatic distress.

A significant proportion of students developed peritraumatic dissociation and high level stressed emotional symptoms. The younger Gen $\mathrm{Z}$ appeared to be less resilient. Specifically, younger students reported higher peritraumatic dissociative experience and lower e-learning affinity levels. Among academic education fields, Human and Social Sciences students reported higher extroversion personality trait, isolation stress, and dysfunctional coronavirus anxiety symptoms. Socio-demographic characteristics, student residential status (on-campus vs. off-campus students), study area, and the geographical location of the university did not exert a significant effect on stress.

Moreover, we wanted to examine the relationship between emotional regulation, personality traits, e-learning affinity and risk for mental health. High level of conscientiousness (tendency to be persistent and determined in achieving personal goals) and acceptability (the tendency to be sympathetic, cooperative, and accommodating) appear to have acted as a protective factor against peritraumatic dissociation, relationship and academic life, and isolation stress. In contrast, low level of emotional stability (the tendency to be resilient and do not react easily to stress) is correlated to fear of coronavirus contagion, and high level of openness (the tendency to be interested in having experiences that expand their minds) is correlated to anxiety and relationship and academic life stress.

Another interesting finding pertains to the effect of e-learning affinity on emotional regulation. Specifically, higher levels of e-learning affinity may weaken peritraumatic dissociation, dysfunctional coronavirus anxiety, relationship and academic life and isolation stress. 
The impact of the prolonged academic e-learning on the mental health of academic community was strong and unmanageable by themselves; individual resources did not help undergraduate students to overcome the distress. Gen $Z$ appeared to be exposed to mental distress and needed help. Our findings provided details for protective and predictive risk factors.

\section{Conclusions}

The present findings suggest that, because coronavirus distress is a significant predictor of mental health through the mediating effect of e-learning affinity and personality traits, health care professionals should design and implement interventions and programmes that focus on COVID-19peritraumatic dissociation and stress. Health care professionals are encouraged to implement psychological support interventions that strengthen one's ability to manage stressful situations and reinforce their status as a digital native.

\section{References}

Adedoyin O, \& Soykan E. (2020). Covid-19 pandemic and online learning: the challenges and opportunities. Interactive Learning Environments.

Appolloni A, Colasanti N, Fantauzzi C, Fiorani G, \& Frondizi R. (2021). Distance Learning as a Resilience Strategy during Covid-19: An Analysis of the Italian Context. Sustainability, 13(3):1388.

Dimock, M. Defining generations: Where Millennials end and Generation Z begins. January 7, 2019, https://www.pewresearch.org/fact-tank/2019/01/17/where-millennials-end-and-generation-zbegins/

Guido, G, Peluso, AM, Capestro, M, \& Miglietta, M (2015). An Italian version of the 10-item Big Five Inventory: an application to hedonic and utilitarian shopping values. Personality and Individual Differences, 76, 135-140.

https://opendatadpc.maps.arcgis.com/apps/opsdashboard/index.html\#/b0c68bce2cce478eaac82fe38d4138 b1

Lee, S (2020). Coronavirus Anxiety Scale: A brief mental health screener for COVID-19 related anxiety. Death Studies, 44:7, 393-401.

Marmar, C., Weiss, DS, \& Metzler, TJ (1997). The Peritraumatic Dissociative Experiences Questionnaire. In J. P. Wilson \& T. M. Keane (Eds.), Assessing psychological trauma and PTSD (pp. 412-428): Guildford Press.

Quintiliani L, Sisto A, Vicinanza F, Curcio G, \& Tambone V. (2021) Resilience and psychological impact on Italian university students during COVID-19 pandemic. Distance learning and health, Psychology, Health \& Medicine.

Zurlo MC, Cattaneo Della Volta MF; \& Vallone F (2020). COVID-19 Student Stress Questionnaire: Development and Validation of a Questionnaire to Evaluate Students' Stressors Related to the Coronavirus Pandemic Lockdown. Front. Psychol. 11:576758. 